\title{
基于方向调制的物理层安全无线传输原理、关键 技术与未来应用
}

\author{
束锋 $1,2,3,4$ ，吴肖敏 ${ }^{1} ， e^{\text {肖虎 }}{ }^{3}$ ，陆锦辉 $1^{*}$ ，胡锦松 ${ }^{1}$ ，朱伟 ${ }^{1} ，$ 余海 $^{1}$, \\ 许正文 ${ }^{2}$, 陈日清 ${ }^{4}$ \\ 1. 南京理工大学电子工程与光电技术学院, 南京 210094 \\ 2. 电子科技集团公司第二十二研究所电波环境特性及模块化技术国防科技重点实验室，青岛 266107 \\ 3. 东南大学移动通信国家重点实验室, 南京 210096 \\ 4. 福建农林大学计算机与信息学院, 福州 350002 \\ * 通信作者. E-mail: lujinhui_ee_1962@163.com
}

收稿日期: 2016-12-09; 接受日期: 2017-05-17; 网络出版日期: 2017-08-23

国家自然科学基金 (批准号: 61271230,61472190,61501238)、中央高校基本科研业务费专项资金 (批准号: 30916011205)、电子科 技集团公司第二十二研究所电波环境特性及模块化技术国防科技重点实验室开放课题 (批准号: 201500013) 和东南大学移动通信国 家重点实验室开放课题 (批准号: 2013D02) 资助项目

\begin{abstract}
摘要 方向调制作为一种先进的物理层安全传输技术, 将波束成形技术和人工噪声结合进一步提高 通信系统的安全性能. 不同于传统的波束成形技术, 方向调制技术能够实现信号的方向性, 保证期望 方向信号的安全传输，同时干扰非期望方向上信号的星座图。该文对方向调制技术的概念和特点进 行讨论, 系统地阐述方向调制合成方法与性能度量等关键技术, 总结归纳方向调制的研究现状, 对其 未来的研究方向和亟待解决的关键问题进行深入探讨与分析, 并提供可行的解决思路. 为了克服传 统方向调制仅存在角度依赖性的问题, 引入频率分集, 提出了精准无线传输新概念, 能实现给定任意 角度与距离维的精准安全无线传输.
\end{abstract}

关键词 方向调制, 物理层安全, 人工噪声, 波束成形, 精准通信

\section{1 引言}

近些年来, 随着移动互联网应用和业务的爆发增长, 移动通信和无线网络依然延续其 20 世纪末 的快速发展态势. 移动通信和无线网络在人们的日常生活中发挥着不可替代的作用, 并对社会的进步 产生重大的影响. 然而, 由于无线通信与生俱来的广播特性, 电磁波向四周传播, 信息容易被未经授权 的接收机拦截或窃听 ${ }^{[1]}$. 因此, 无线通信物理层安全问题最近引起了学术界和工业界广泛的关注, 成

\footnotetext{
引用格式: 束锋, 吴肖敏, 尤肖虎, 等. 基于方向调制的物理层安全无线传输原理、关键技术与未来应用. 中国科学: 信息科学, 2017, 47: 1209-1225, doi: 10.1360/N112016-00280

Shu F, Wu X M, You X H, et al. Directional modulation-based secure wireless transmission: basic principles, key techniques, and applications (in Chinese). Sci Sin Inform, 2017, 47: 1209-1225, doi: 10.1360/N112016-00280
} 
为无线通信领域研究热点方向, 将在未来无线通信中发挥至关重要的作用. 传统的解决方案主要是采 用上层加密技术 ${ }^{[2]}$, 但加密系统密钥仍然需要信道传递, 其安全协议仍然难以保障. 为了获得更好的 安全性, 处于协议层最底层的物理层进入了人们的视野 ${ }^{[3,4]}$. 物理层安全的研究源于 Wyner ${ }^{[5]}$ 提出的 Wire-tap 模型, 后来学者提出利用人为噪声的方法来有效控制主信道和窃听信道的优劣, 将上层加密 技术与物理层安全技术相结合, 可以实现无线通信安全的双重保障. 方向调制作为一种有效的物理层 传输技术, 最近引起相当的研究兴趣, 其基本思想: 通过波束成形和人为噪声等处理手段, 在期望方向, 接收到的信号星座图与发射基带星座图保持一致, 在其他窃听方向接收的信号星座产生畸变或被噪声 严重污染, 于是期望接收机可以正确地解调接收信号, 而窃听接收机误码性能恶化而无法正常解调出 有用信息. 据我们所知, 方向调制是一种主要适用于远场无线通信的物理层安全技术, 因为近场的方 向性不易控制.

目前方向调制 (directional modulation, DM) 的方法主要分为两类, 一种是利用射频端元器件的组 合来实现, 另外一种侧重于基带信号的算法设计来实现. 文献 [6,7] 中, 在射频域的调制是通过耦合到 一个驱动天线的寄生天线阵元来实现. 其提出的近场天线直接调制技术的发射信号是一种由多个波 束在空间中干涉形成方向调制信号的射频调制技术. 文献 [8] 提出了一种基于相控阵的方向调制技术, 其调制过程需要发生在射频阶段, 而不是常规的基带调制, 该技术主要缺陷: 只考虑了在期望方位合 成基带数字调制信号星座图而没有考虑在非期望方位星座图的畸变程度. 文献 [9] 基于文献 [8] 中的 理论基础设计实现了基于相控阵的物理层安全通信系统. 文献 [10] 中基于切换天线的可重构阵列, 提 出了一种与文献 [8] 中相似的技术, 采用未经调制的载波直接激励可重构阵, 通过发射天线的切换重 构发射阵列, 在期望方向综合出基带数字调制信号, 在期望方位天线的一种重构状态对应基带调制信 号星座图中的一个星座点, 发射阵列通过天线发射状态的重构传输信息. 文献 [11] 提出了一种基于方 向调制的双波束扩频信号, 发射信号不仅与使用的扩频序列有关, 而且与接收机相对于发射机的空间 方位信息有关, 是一种扩频序列和空间方位信息共同调制的空间扩频信号. 文献 [12] 提出了一种运用 多目标函数遗传算法的方向调制信号, 根据星座点之间欧氏距离与误码性能之间的关系, 采用多目标 函数优化获得相移器相移值, 使得发射信号星座图在期望方位与基带调制信号相同, 而在其他方位产 生最大程度的畸变. 然而, 上述方案受限于有限的天线阵列排布结构、高速 RF 开关和高精度的相移 器, 实现的复杂度和成本较高. 此外信号星座图变化范围较小, 窃听者可以通过观察发现其变化规律, 从而破译.

文献 [13] 提出在基带端合成方向调制信号, 通过加入与期望方向正交的人工噪声 (articial noise, $\mathrm{AN})$, 信号的星座图保持动态变化, 从而使窃听者不能追踪信号星座图轨迹变化的规律, 从而很难对有 用信息跟踪和解码. 文中将方向调制合成算法分为两类: 第一类称之为 “静态” 算法, 所产生的天线 图对于任何选定的星座点是不改变的, 即如果我们选择发送单一的信号, 生成的信号轨迹图不会改变; 第二类称之为 “动态” 算法, 相同的信号会产生不同的轨迹图, 文献 [14] 在文献 [13] 的基础上推广到 多波束 DM 系统. 文献 [15] 利用奇异值分解 (singular value decomposition, SVD) 的方法, 提出了在两 径环境下信息安全传输的方案, 缩小了错误自由区. 文献 [16] 提出了多波束方向调制的发射方案, 该 技术利用最小范数推导出阵列加权系数, 为多用户多输入多输出 (multi-input multi-output, MIMO) 的 安全传输提供了通信连接, 同时系统的传输容量会随着天线阵元的数量增长. 文献 [17] 在无线干扰网 络中做了一些基础性的工作, 利用干扰对其技术提出了集中新颖的物理层安全通信的方法.

但以上所提方案均假定已知完美的方向角信息, 而在实际通信场景中, 方向角需要通过 MUSIC 以及 Capon 等空间谱估计算法来估计, 估计会产生误差, 从而恶化期望方向上接收性能. 文献 $[18,19]$ 分别针对不同应用场景提出稳健的方向调制合成方法. 文献 [18] 针对单用户系统, 基于角度误差统计 


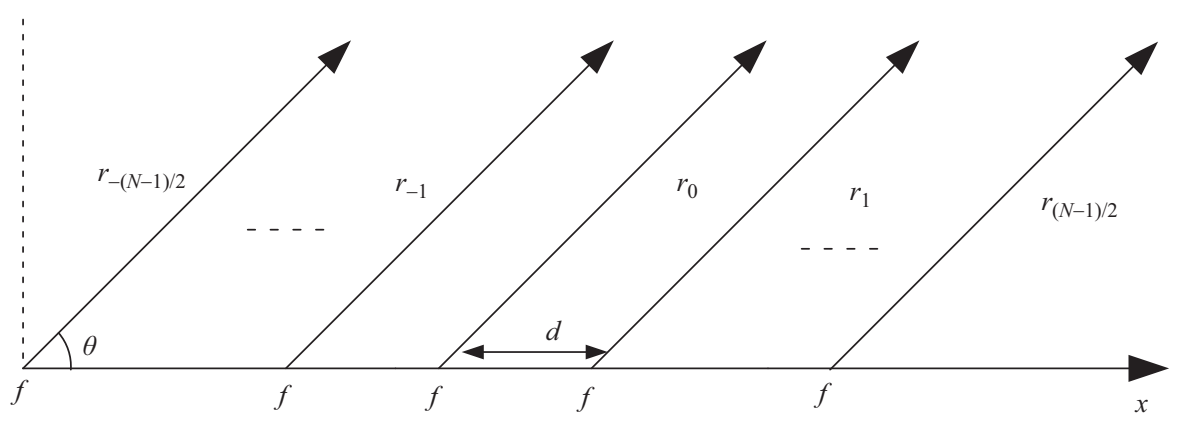

图 1 方向调制天线阵列图

Figure 1 Illustration of directional modulation antenna array

特性和最小均方误差概念设计优化, 以减小估计误差对期望用户带来的影响. 文献 [19] 针对多波束广 播系统, 采用截断 Gauss 分布, 基于信泄噪比和条件期望推导角度误差下的波束成形向量和人工噪声, 实现信息稳健的安全传输.

方向调制与传统物理层安全技术的区别: 传统的物理层安全主要针对无线衰落信道, 由于传输多 径与 Doppler 扩张等因素影响, 无线信道表现出时变与衰落特性, 此场景下安全的波束成形与人工噪 声的设计充分挖掘合法通信双方的衰落信道矩阵特征空间/奇异值向量空间等期望信道空间, 使得隐 私信息尽可能在期望信道空间中可靠传输, 而人工噪声尽量在期望空间零空间传输, 从而导致窃听者 难以获取隐私信息. 而方向调制主要适用于无衰落 Gauss 白噪声信道, 信道导向向量带有方向角信息, 有用信号波束成形向量通过期望用户导向向量进行设计, 人工噪声在期望方向导向向量零空间、窃听 信道导向向量空间或者两者空间交集上传输. 不同于衰落信道的时变特性, 若发射机与接收机保持静 止时, 方向调制导向向量表现出静态稳定性. 但是, 方向调制与传统的物理层安全技术均属无线物理 层安全的范畴.

本文结构安排如下: 第 2 节介绍方向调制技术的基本原理和模型. 第 3 节讨论方向调制系统合成 与性能度量等关键技术. 第 4 节介绍精准无线通信的基本概念与理论. 第 5 节对未来函待解决的问题 进行探讨和分析. 第 6 节总结全文.

\section{2 方向调制基本原理与系统模型}

图 1 所示为用于方向调制的均匀各向同性线性阵列示意图. 该阵列由 $N$ 个距离为半个波长的阵 元组成. 在该系统中, $M$ 路独立的数据流 $\left\{x_{m}\right\}_{m=1}^{M}$ 分别发送到 $M$ 个期望接收机方向, 方向角分别为 $\left\{\theta_{d_{1}}, \theta_{d_{2}}, \ldots, \theta_{d_{M}}\right\}$. 此外, 假设存在 $J$ 个窃听方向, 分别为 $\left\{\theta_{u_{1}}, \theta_{u_{2}}, \ldots, \theta_{u_{J}}\right\}$. 令调制第 $m$ 个用户的 第 $k$ 个符号为 $x_{m k}$, 并且满足 $\mathrm{E}\left\{x_{m k}^{*} x_{m k}\right\}=1$. 发射机在发射端同时发射有用信号和人工噪声, 第 $k$ 个符号的传输向量表示为

$$
\boldsymbol{s}_{k}=\underbrace{\beta_{1} \sqrt{P_{\mathrm{s}}} \sum_{m=1}^{M} \boldsymbol{v}_{m} x_{m k}}_{\text {Confidential messages }}+\underbrace{\beta_{2} \sqrt{P_{\mathrm{s}}} \boldsymbol{W} \boldsymbol{z}}_{\mathrm{AN}},
$$

式中, $P_{\mathrm{s}}$ 表示总的发射功率, $\boldsymbol{v}_{m}$ 表示第 $m$ 路数据流的 $N \times 1$ 的波束成形向量, 且 $\boldsymbol{v}_{m}^{\mathrm{H}} \boldsymbol{v}_{m}=1, \beta_{1}$ 和 $\beta_{2}$ 分别表示有用信号和人工噪声的功率分配系数, 并满足 $\beta_{1}^{2}+\beta_{2}^{2}=1, \boldsymbol{z} \in \mathcal{C}^{N \times 1}$ 是概率分布为 
$\mathcal{C N}\left(0, \boldsymbol{I}_{N}\right)$ 的向量, $\boldsymbol{W}$ 为正交投影矩阵, 将人工噪声投射到期望方向导向向量的零空间. 发射信号数 据流 $s$ 通过无线信道传输, 沿方向角 $\theta$ 接收到的第 $k$ 个数据表示为

$$
y_{k}(\theta)=\boldsymbol{h}^{\mathrm{H}}(\theta) \boldsymbol{s}_{k}+\omega_{k},
$$

式中, $\omega_{k}$ 为加性 Gauss 白噪声 $(\mathrm{AWGN})$, 其分布为 $\mathcal{C N}\left(0, \sigma_{\omega_{k}}^{2}\right)$, 其归一化导向向量表示为

$$
\boldsymbol{h}(\theta)=\frac{1}{\sqrt{N}}[\underbrace{\mathrm{e}^{\mathrm{j} 2 \pi \varphi_{\theta}(1)}}_{h_{1}(\theta)}, \ldots, \underbrace{\mathrm{e}^{\mathrm{j} 2 \pi \varphi_{\theta}(n)}}_{h_{n}(\theta)}, \ldots, \underbrace{\mathrm{j} 2 \pi \varphi_{\theta}(N)}_{h_{N}(\theta)}]^{\mathrm{T}},
$$

其中 $\varphi_{\theta}(n)$ 定义为

$$
\varphi_{\theta}(n) \triangleq \frac{(n-(N+1) / 2) d \cos \theta}{\lambda} \quad(n=1,2, \ldots, N),
$$

式 (4) 中, $d$ 为阵元间距, $\lambda$ 为载波波长. 根据式 (2), 第 $m$ 个期望用户的接收信号数据流表示为

$$
\begin{aligned}
y\left(\theta_{d_{m}}\right) & =\boldsymbol{h}^{\mathrm{H}}\left(\theta_{d_{m}}\right) \boldsymbol{s}+\omega_{d_{m}} \\
& =\underbrace{\beta_{1} \sqrt{P_{\mathrm{s}}} \boldsymbol{h}^{\mathrm{H}}\left(\theta_{d_{m}}\right) \boldsymbol{v}_{m} x_{m}}_{\text {Useful signals }}+\underbrace{\beta_{1} \sqrt{P_{\mathrm{s}}} \boldsymbol{h}^{\mathrm{H}}\left(\theta_{d_{m}}\right) \sum_{i=1, i \neq m}^{M} \boldsymbol{v}_{i} x_{i}}_{\text {Interference from other users }}+\underbrace{\beta_{2} \sqrt{P_{\mathrm{s}}} \boldsymbol{h}^{\mathrm{H}}\left(\theta_{d_{m}}\right) \boldsymbol{W} \boldsymbol{z}}_{\text {AN }}+\underbrace{\omega_{d_{m}}}_{\text {AWGN }},
\end{aligned}
$$

其中, 等式右边第一项为第 $m$ 个期望用户接收到的有用信号; 第二项为用户之间的相互干扰, 由于对 每个期望用户数据的波束成形向量分别进行设计, $\boldsymbol{h}^{\mathrm{H}}\left(\theta_{d_{m}}\right) \boldsymbol{v}_{i} x_{i}$ 即为第 $i$ 个期望用户数据流对第 $m$ 个 期望用户接收数据产生的干扰; 第三项为接收到的人工噪声, 设计投影矩阵 $\boldsymbol{W}$, 使得期望方向接收到 的人工噪声几乎为零, 从而最小化人工噪声对期望方向的影响; 最后一项为分布满足 $\mathcal{C N}\left(0, \sigma_{d_{m}}^{2}\right)$ 的接 收噪声. 第 $j$ 个窃听者接收信号表示为

$$
\begin{aligned}
y\left(\theta_{u_{j}}\right) & =\boldsymbol{h}^{\mathrm{H}}\left(\theta_{u_{j}}\right) \boldsymbol{s}+\omega_{u_{j}} \\
& =\underbrace{\beta_{1} \sqrt{P_{\mathrm{s}}} \boldsymbol{h}^{\mathrm{H}}\left(\theta_{u_{j}}\right) \sum_{m=1}^{M} \boldsymbol{v}_{m} x_{m}}_{\text {Confidential messages }}+\underbrace{\beta_{2} \sqrt{P_{\mathrm{s}}} \boldsymbol{h}^{\mathrm{H}}\left(\theta_{u_{j}}\right) \boldsymbol{W} \boldsymbol{z}}_{\text {AN }}+\underbrace{\omega_{u_{j}}}_{\text {AWGN }} .
\end{aligned}
$$

上式中, 等式右边第一项为第 $j$ 个窃听者窃听到的保密信息, 通过对每路用户数据波束成形向量 $\boldsymbol{v}_{m}$ 的设计, 以最小化保密信息到达穷听者的功率, 减小被钬听的威胁; 第二项为窃听者接收到的人工噪 声, 最大化 $\boldsymbol{h}^{\mathrm{H}}\left(\theta_{u_{j}}\right) \boldsymbol{W} \boldsymbol{z}$ 对窃听者的污染, 使得窃听者接收到人工噪声的功率最大化, 严重干扰窃听者 对保密信息的接收; 最后一项为接收噪声, 其概率分布满足 $\mathcal{C N}\left(0, \sigma_{u_{j}}^{2}\right)$. 利用波束成形算法和人工噪声 的动态随机性防御窃听者对有用信息的窃取, 为通信系统的安全提供保障.

\section{3 方向调制合成方法与性能测度}

通信系统安全性能的提高, 方向调制合成技术起着至关重要的作用, 主要包括系统的合成方法与 发射功率分配. 合理设计有用信号波束成形向量和人工噪声, 以求在最小化影响期望用户性能的条件 下, 极大地恶化窃听者的性能. 同时为进一步探讨验证方向调制系统的系统性能优势, 下面对性能测 度提供了合适的度量标准. 


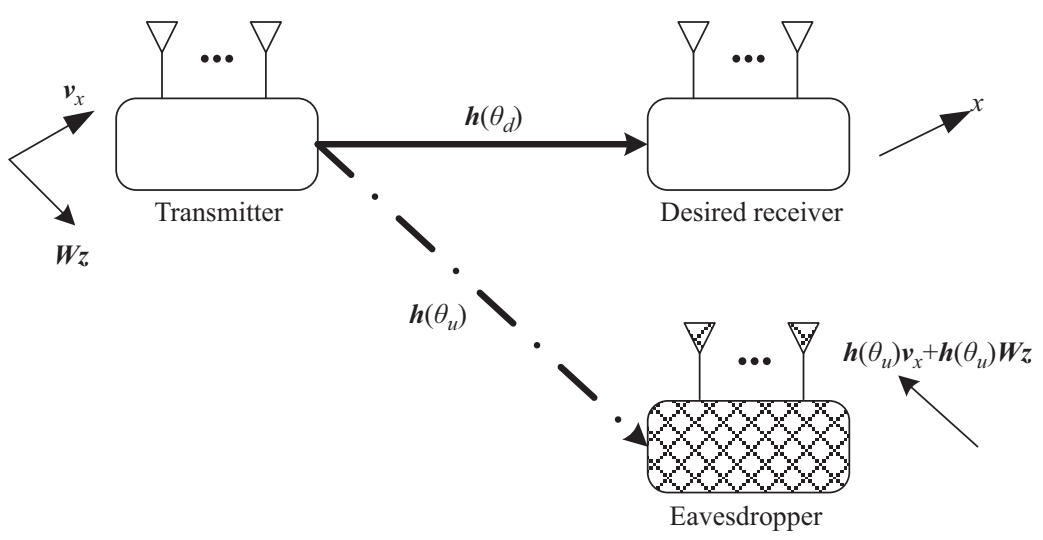

图 2 单期望方向系统

Figure 2 Single-beam directional modulation system

\section{1 合成方法}

本文对方向调制系统的合成主要考虑有用信号的波束成形和人工噪声的设计, 方向调制的主要特 点在于其发射波束的角度依赖性, 方向角信息的准确性直接影响算法的性能, 在实际通信系统中, 对 于方向角的估计不可避免会产生误差, 下面将分两种情况介绍方向调制的合成算法: 完美方向角和非 完美方向角.

\subsection{1 完美方向角}

在方向角信息完美已知的情况下, 本节主要分为单期望方向系统和多期望方向系统两种应用场景.

单期望方向系统. 图 2 为单期望方向调制系统图, 即 $M=1$, 利用天线波束来提供方向的安全 性. 根据式 (5), 在单期望用户系统中, 期望接收机方向角表示为 $\theta_{d}$, 窃听方向角可表示为 $\theta_{u}$. 期望方 向的接收信号表示为

$$
\begin{aligned}
y\left(\theta_{d}\right) & =\boldsymbol{h}^{\mathrm{H}}\left(\theta_{d}\right) \boldsymbol{s}+\omega_{d} \\
& =\beta_{1} \sqrt{P_{\mathrm{s}}} \boldsymbol{h}^{\mathrm{H}}\left(\theta_{d}\right) \boldsymbol{v} x+\beta_{2} \sqrt{P_{\mathrm{s}}} \boldsymbol{h}^{\mathrm{H}}\left(\theta_{d}\right) \boldsymbol{W} \boldsymbol{z}+\omega_{d},
\end{aligned}
$$

非期望方向的接收信号表示为

$$
\begin{aligned}
y\left(\theta_{u}\right) & =\boldsymbol{h}^{\mathrm{H}}\left(\theta_{u}\right) \mathbf{s}+\omega_{u} \\
& =\beta_{1} \sqrt{P_{\mathrm{s}}} \boldsymbol{h}^{\mathrm{H}}\left(\theta_{u}\right) \boldsymbol{v} x+\beta_{2} \sqrt{P_{\mathrm{s}}} \boldsymbol{h}^{\mathrm{H}}\left(\theta_{u}\right) \boldsymbol{W} \boldsymbol{z}+\omega_{u} .
\end{aligned}
$$

不同于传统的波束成形, DM 的信号在基带合成, 有用信号的激励信号向量对应于期望方向的导 向向量, 对于人工噪声的设计, 通过设计正交投影矩阵 $\boldsymbol{W}$, 将人工噪声投影到期望方向导向向量的零 空间, 即

$$
\begin{gathered}
\boldsymbol{v}=\boldsymbol{h}\left(\theta_{d}\right), \\
\boldsymbol{W}=\boldsymbol{I}_{N}-\boldsymbol{h}\left(\theta_{d}\right)\left[\boldsymbol{h}^{\mathrm{H}}\left(\theta_{d}\right) \boldsymbol{h}\left(\theta_{d}\right)\right]^{-1} \boldsymbol{h}^{\mathrm{H}}\left(\theta_{d}\right),
\end{gathered}
$$

其中

$$
\boldsymbol{h}^{\mathrm{H}}\left(\theta_{d}\right) \boldsymbol{W}=\boldsymbol{h}^{\mathrm{H}}\left(\theta_{d}\right)\left\{\boldsymbol{I}_{N}-\boldsymbol{h}\left(\theta_{d}\right)\left[\boldsymbol{h}^{\mathrm{H}}\left(\theta_{d}\right) \boldsymbol{h}\left(\theta_{d}\right)\right]^{-1} \boldsymbol{h}^{\mathrm{H}}\left(\theta_{d}\right)\right\}=\mathbf{0} .
$$


上式中 (. $)^{-1}$ 表示矩阵逆运算. 激励信号波束成形和人工噪声的设计带来一定的优势: (1) 期望方向有 用信号的接收不受人工噪声的影响; (2) 由于人工噪声的动态变化, 窃听者难以寻找变化规律来窃取有 用信息或者接收的有用信号由于人工噪声的存在而被严重扰乱.

多期望方向系统. 在该系统中, 存在 $M$ 个期望接收机和 $J$ 个非期望接收机. 期望方向的导向向 量形成联合矩阵, 所有的期望方向看作一个整体用户，不考虑期望接收机相互之间的信息干扰，类似 地, 窃听机亦看作为整体. 在多期望方向广播系统中, 期望用户方向群表示为 $\Theta_{d}=\left\{\theta_{d_{1}}, \theta_{d_{2}}, \ldots, \theta_{d_{M}}\right\}$, 非期望用户方向群表示为 $\Theta_{u}=\left\{\theta_{u_{1}}, \theta_{u_{2}}, \ldots, \theta_{u_{J}}\right\}$. 根据式 (5), 期望方向的接收信号表示为

$$
\begin{aligned}
\boldsymbol{y}\left(\Theta_{d}\right) & =\boldsymbol{H}^{\mathrm{H}}\left(\Theta_{d}\right) \boldsymbol{s}+\varpi_{d} \\
& =\beta_{1} \sqrt{P_{\mathrm{s}}} \boldsymbol{H}^{\mathrm{H}}\left(\Theta_{d}\right) \boldsymbol{v} x+\beta_{2} \sqrt{P_{\mathrm{s}}} \boldsymbol{H}^{\mathrm{H}}\left(\Theta_{d}\right) \boldsymbol{W} \boldsymbol{z}+\varpi_{d},
\end{aligned}
$$

非期望方向的接收信号表示为

$$
\begin{aligned}
\boldsymbol{y}\left(\Theta_{u}\right) & =\boldsymbol{H}^{\mathrm{H}}\left(\Theta_{u}\right) \boldsymbol{s}+\varpi_{u} \\
& =\beta_{1} \sqrt{P_{\mathrm{s}}} \boldsymbol{H}^{\mathrm{H}}\left(\Theta_{u}\right) \boldsymbol{v} x+\beta_{2} \sqrt{P_{\mathrm{s}}} \boldsymbol{H}^{\mathrm{H}}\left(\Theta_{u}\right) \boldsymbol{W} \boldsymbol{z}+\varpi_{u},
\end{aligned}
$$

式中, $\varpi_{d}$ 和 $\varpi_{u}$ 分别表示 $M \times 1$ 和 $J \times 1$ 的复 Gauss 噪声向量, 且 $\varpi_{d} \sim \mathcal{C N}\left(0, \sigma_{d}^{2} \boldsymbol{I}_{N}\right), \varpi_{u} \sim$ $\mathcal{C N}\left(0, \sigma_{u}^{2} \boldsymbol{I}_{N}\right) \cdot \boldsymbol{H}^{\mathrm{H}}\left(\Theta_{d}\right)$ 和 $\boldsymbol{H}^{\mathrm{H}}\left(\Theta_{u}\right)$ 分别表示期望方向和窃听方向的联合信道矩阵, 令

$$
\begin{aligned}
\boldsymbol{H}\left(\Theta_{d}\right) & =\left[\boldsymbol{h}\left(\theta_{d_{1}}\right), \boldsymbol{h}\left(\theta_{d_{2}}\right), \ldots, \boldsymbol{h}\left(\theta_{d_{M}}\right)\right], \\
\boldsymbol{H}\left(\Theta_{u}\right) & =\left[\boldsymbol{h}\left(\theta_{u_{1}}\right), \boldsymbol{h}\left(\theta_{u_{2}}\right), \ldots, \boldsymbol{h}\left(\theta_{u_{J}}\right)\right] .
\end{aligned}
$$

如图 3 所示, 发射机按照设定的功率分配参数, 在发射端同时发射出一定功率的有用信号和人工噪声 干扰信号. 通过设计有用信号波束成形向量 (即 $\boldsymbol{v}$ ) 和人工噪声向量 (即 $\boldsymbol{W} \boldsymbol{z}$ ). 在期望方向上, 最大化 有用信号的接收功率, 使其与泄露到窃听方向的有用信号和接收噪声的功率比值最大化, 从而保证期 望方向用户对有用信号的接收

$$
\max _{\boldsymbol{v}} \operatorname{SLNR}(\boldsymbol{v}) \quad \text { s.t. } \boldsymbol{v}^{\mathrm{H}} \boldsymbol{v}=1
$$

其中

$$
\operatorname{SLNR}(\boldsymbol{v}) \triangleq \frac{\beta_{1}^{2} P_{\mathrm{s}} \operatorname{tr}\left\{\boldsymbol{v}^{\mathrm{H}} \boldsymbol{H}\left(\Theta_{d}\right) \boldsymbol{H}^{\mathrm{H}}\left(\Theta_{d}\right) \boldsymbol{v}\right\}}{\operatorname{tr}\left\{\boldsymbol{v}^{\mathrm{H}}\left(\beta_{1}^{2} P_{\mathrm{s}} \boldsymbol{H}\left(\Theta_{u}\right) \boldsymbol{H}^{\mathrm{H}}\left(\Theta_{u}\right)+\sigma_{d}^{2} \boldsymbol{I}_{N}\right) \boldsymbol{v}\right\}} .
$$

引入泄露的概念, 设计出有用信号的波束成形向量, 对于人工噪声投影矩阵 $\boldsymbol{W}$ 的设计, 最大化期望方 向的平均接收信噪比

其中

$$
\max _{\boldsymbol{W}} \operatorname{SANR}(\boldsymbol{W}, \boldsymbol{v})
$$

$$
\operatorname{SANR}(\boldsymbol{W}, \boldsymbol{v}) \triangleq \frac{\beta_{1}^{2} P_{\mathrm{s}} \operatorname{tr}\left\{\boldsymbol{H}^{\mathrm{H}}\left(\Theta_{d}\right) \boldsymbol{v} \boldsymbol{v}^{\mathrm{H}} \boldsymbol{H}\left(\Theta_{d}\right)\right\}}{\beta_{2}^{2} P_{\mathrm{s}} \operatorname{tr}\left\{\boldsymbol{H}^{\mathrm{H}}\left(\Theta_{d}\right) \boldsymbol{W} \boldsymbol{W}^{\mathrm{H}} \boldsymbol{H}\left(\Theta_{d}\right)\right\}+\sigma_{d}^{2}},
$$

式中, 随着分母当中的第一项不断减小, 平均接收信噪比将逐渐增大, 当该项为零时, SANR 将达到最 大值. 基于信泄噪比和信干噪比两种准则的理论设计有用信号的波束成形向量与人工噪声向量 ${ }^{20]}$.

在多期望方向广播系统中, 存在窃听者方向角信息已知和未知两种情况. 窃听者方向角信息已知, 发射机根据完美的信道状态信息, 对窃听者进行人工干扰, 同时保证期望用户对有用信号的接收, 一旦 苆听方向角信息未知, 发射机无法获取准确的窃听信道状态信息. 对于期望方向角主瓣之外的所有的 方向角度, 都认为是潜在的窃听者, 人工噪声的干扰主要集中在主瓣外的角度, 以减小有用信息被窃听 的威胁. 


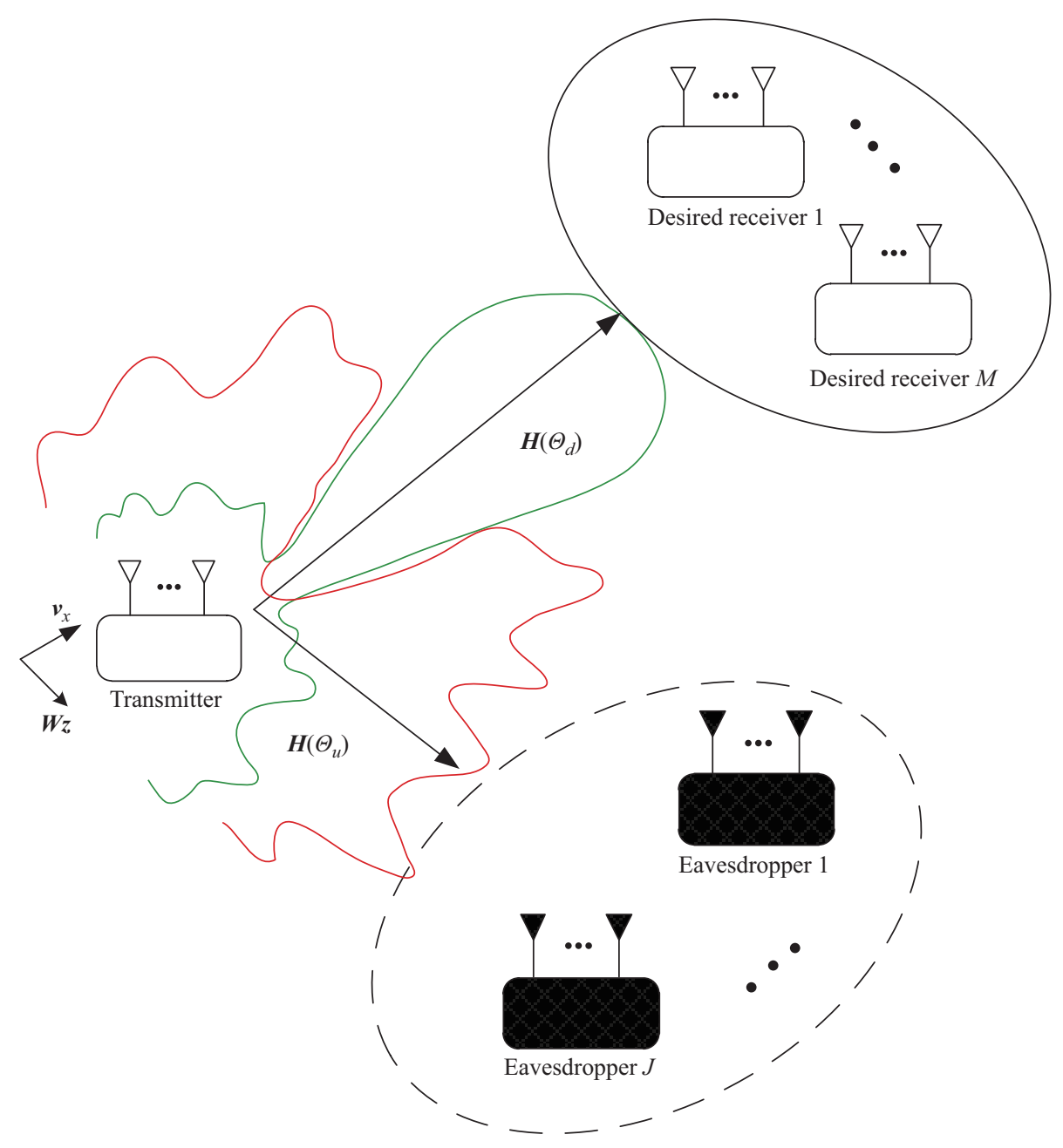

图 3 (网络版彩图) 多期望方向系统

Figure 3 (Color online) Multi-beam broadcasting directional modulation system

\subsection{2 非完美方向角}

一般地, 为了实现方向调制, 发射机需要提前知道期望用户方向角的信息. 但在实际应用场景, 无 论是 GPS 定位服务, 还是传统的估计算法, 如 Capon, MUSIC 和 ESPRIT 等得到角度信息 ${ }^{[21]}$, 都会 带来一定的角度误差. 为了减少角度误差带来的影响, 基于角度误差统计特性和条件期望概念设计优 化有用信号波束成形向量与人工噪声投影矩阵, 从而设计稳健的方向调制合成算法.

单期望方向系统. 在单期望方向系统中, 由于估计误差, 期望方向方向角为 $\hat{\theta}_{d}=\theta_{d}+\Delta \theta_{d}\left(\hat{\theta}_{d}\right.$ 为 估计角, $\Delta \theta_{d}$ 为角度误差). 文献 [18] 中, 角度误差概率分布遵循均匀分布, 提出了基于条件期望和最 小均方误差 (minimum mean square error, MMSE) 的稳健方向调制合成算法. 引入误差分布和条件期 望的概念, 推导期望方向导向向量的期望值, 计算投影矩阵.

尽管传统的估计算法能够实现角度的估计, 但方向角估计存在的极小的不确定性将会引起激励信 号向量与导向向量不匹配, 从而严重影响期望方向的接收性能. 考虑到方向角的估计误差, 通过条件 
MMSE 建立优化目标函数

$$
\min _{\boldsymbol{v}} \mathrm{E}_{\hat{\theta}_{d}}\left[\left\|y\left(\hat{\theta}_{d}\right)-\beta_{1} \sqrt{P_{\mathrm{s}}} x\right\|^{2}\right] \quad \text { s.t. } \boldsymbol{v}^{\mathrm{H}} \boldsymbol{v} \leqslant 1,
$$

$\mathrm{E}[\cdot]$ 为期望运算, 对目标函数求导运算, 设计优化激励信号向量 $\boldsymbol{v}$, 实现扰动下方向调制系统的设计.

多期望方向系统. 由于信道噪声和干扰的影响, 角度测量误差会直接影响有用信号和人工噪声 向量的设计. 文献 [19] 考虑两种场景: 非完美窃听方向和未知窃听方向, 其中期望方向角和窃听方向 角的误差均为独立同分布, 概率分布为截断 Gauss 分布. 在非完美窃听方向情况下, 重新定义条件信 泄噪比 (conditional signal to leakage-and-noise ratio, CSLNR)

$$
\max _{\boldsymbol{v}} \operatorname{CSLNR}(\boldsymbol{v}) \quad \text { s.t. } \boldsymbol{v}^{\mathrm{H}} \boldsymbol{v}=1 .
$$

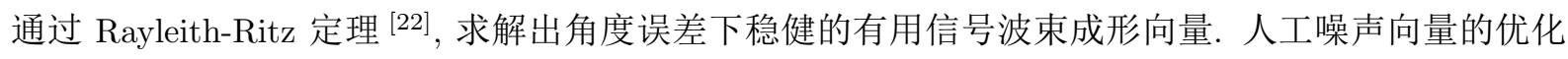
设计则是在给定的误差分布下, 推导出投影矩阵的条件期望表达式.

在窃听方向未知时, 期望方向角的估计误差概率分布仍为截断 Gauss 分布, 期望角主瓣以外均为 潜在的窃听方向, 人工噪声的干扰主要针对窃听方向, 对窃听方向角度范围内的导向向量进行积分. 通 过引入泄露的概念, 最大化条件信泄噪比, 从而得到优化的有用信号的波束成形向量. 人工噪声的设 计则与非完美窃听方向的场景类似.

\section{2 发射功率分配}

方向调制技术将波束成形与人工噪声结合, 发射机在发送有用信号的同时, 发射人工噪声以干扰 窃听机对有用信息的窃取. 对于无线通信系统来说, 采用方向调制技术可以有效地提高系统的性能, 但发射功率的分配也会影响系统的性能, 因而合理分配有用信号和人工噪声的发射功率将提高功率的 利用率, 节约资源. 在文献 [23] 中, 通过最小化发射总功率, 保证期望接收机处的信干噪比达到一定的 约束值, 同时限制非期望接收机的接收信干噪比, 从而联合优化波束成形向量和人工噪声协方差矩阵. 因而, 在发射端同时发送有用信号和人工噪声, 则要对两种信号的发射功率进行合理分配. 有用信号 的发射功率不能过低, 以确保期望方向接收机的接收; 人工噪声的发射功率则要求对非期望方向造成 一定的干扰, 以恶化窃听机性能.

\section{3 性能度量标准}

评价算法好坏的重要指标在于其实现系统的性能优劣. 通信的任务是安全准确地传输信息, 因而 通信系统的主要性能指标包括可靠性和有效性, 接下来将介绍度量方向调制系统的性能标准, 主要包

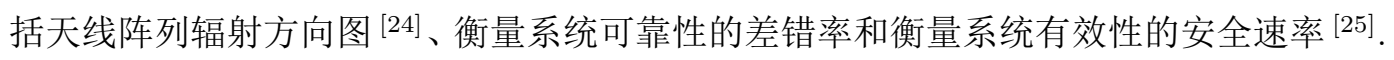

\subsection{1 天线辐射方向图}

方向图, 即天线的辐射电磁场在固定距离上随角度分布的图形, 用功率表示的称为功率方向图, 用 相位表示的称为相位方向图 ${ }^{[13]}$. 如图 4 和 5 所示, 天线阵列辐射方向图很好地反映出信号的功率和 相位信息, 对信号在不同角度的星座图直观地体现. 但由于天线辐射方向图没有考虑信道噪声的影响, 因而从误码率和安全速率指标上进一步的衡量方向调制系统的性能. 


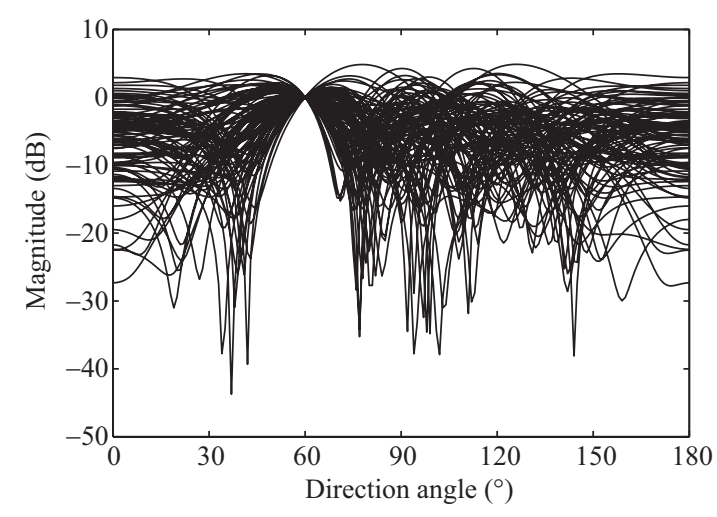

图 4 方向调制阵列幅度图

Figure 4 Power pattern of a DM array for each QPSK (quadrature phase shift keying) symbol

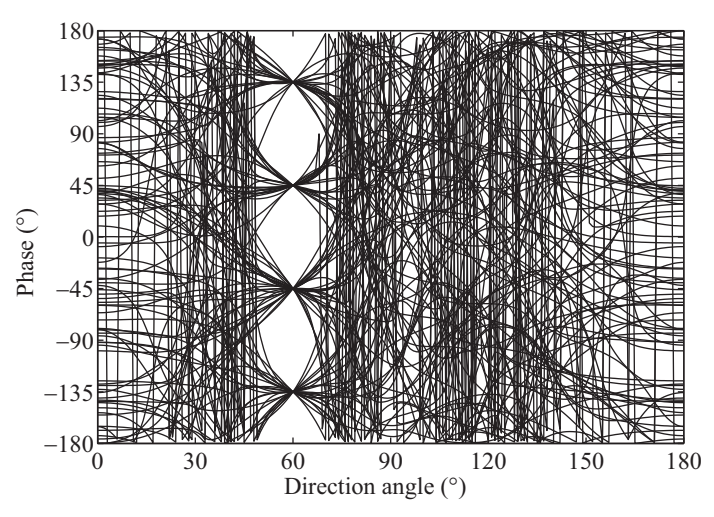

图 5 方向调制阵列相位图

Figure 5 Phase pattern of a DM array for each QPSK symbol

\subsection{2 差错率}

差错率分为误信率和误码率两个指标. 误信率 $\left(\mathrm{ER}_{\mathrm{b}}\right)$, 又称误比特率, 是错误比特数与传输总比 特数之比; 误码率 $\left(\mathrm{ER}_{\mathrm{s}}\right)$ 是错误符号数与传输总符号数之比, 且满足 $\mathrm{ER}_{\mathrm{s}}=\mathrm{ER}_{\mathrm{b}} \cdot \log _{2} K$, 其中 $K$ 为进制数. 这里主要考虑在加性 Gauss 白噪声信道下的 $K$ 元正交幅度调制 (quadrature amplitude modulation, QAM) 的符号错误概率

$$
\mathrm{ER}_{\mathrm{s}, K-\mathrm{QAM}}=1-\left(1-\mathrm{ER}_{\mathrm{s}, \sqrt{K}-\mathrm{PAM}}\right)^{2}
$$

由于

$$
\mathrm{ER}_{\mathrm{s}, \sqrt{K}-\mathrm{PAM}}=2\left(1-\frac{1}{\sqrt{K}}\right) Q\left(\sqrt{\frac{3 \log _{2} K}{K-1} \frac{E_{\mathrm{b}}}{N_{0}}}\right),
$$

式中, $E_{\mathrm{b}} / N_{0}$ 为 $K$-QAM 调制系统的平均比特信噪比, $Q[\cdot]$ 为 Gauss 错误函数

$$
Q(x)=\int_{x}^{\infty} \frac{1}{\sqrt{2 \pi}} \mathrm{e}^{\frac{-y^{2}}{2}} \mathrm{~d} y .
$$

\subsection{3 安全速率和}

通过安全速率指标评价系统的有效性, 可以反映出系统在传输一定信息量是所占用的信道资源, 安全速率越大, 说明 “速度” 越快, 有效性越好. Wyner 指出当主信道在统计概率上要优于窃听信道时, 信息能够得到安全传输, 传输速率的上界称为安全容量, 物理层安全通信的实现是基于主信道的质量 在平均上优于窃听信道 ${ }^{[5]}$. 期望方向上的信道容量被特征化为最大化互信息量, 也就是定义为信道的 最大传输速率, 根据式 (5) 和 (6), 第 $m$ 个期望方向和第 $j$ 个窃听方向关于第 $m$ 路数据流的信息速率 分别为

$$
\begin{gathered}
R_{m}\left(\theta_{d_{m}}\right)=\log _{2}\left(1+\frac{\beta_{1}^{2} P_{\mathrm{s}} \boldsymbol{h}^{\mathrm{H}}\left(\theta_{d_{m}}\right) \boldsymbol{v}_{m} \boldsymbol{v}_{m}^{\mathrm{H}} \boldsymbol{h}\left(\theta_{d_{m}}\right)}{\sigma_{d_{m}}^{2}+\sum_{i=1, i \neq m}^{M} \beta_{1}^{2} P_{\mathrm{s}} \boldsymbol{h}^{\mathrm{H}}\left(\theta_{d_{m}}\right) \boldsymbol{v}_{i} \boldsymbol{v}_{i}^{\mathrm{H}} \boldsymbol{h}\left(\theta_{d_{m}}\right)+\beta_{2}^{2} P_{\mathrm{s}} \boldsymbol{h}^{\mathrm{H}}\left(\theta_{d_{m}}\right) \boldsymbol{W} \boldsymbol{W}^{\mathrm{H}} \boldsymbol{h}\left(\theta_{d_{m}}\right)}\right) \\
R_{m}\left(\theta_{u_{j}}\right)=\log _{2}\left(1+\frac{\beta_{1}^{2} P_{\mathrm{s}} \boldsymbol{h}^{\mathrm{H}}\left(\theta_{u_{j}}\right) \boldsymbol{v}_{m} \boldsymbol{v}_{m}^{\mathrm{H}} \boldsymbol{h}\left(\theta_{u_{j}}\right)}{\sigma_{u_{j}}^{2}+\sum_{i=1, i \neq m}^{M} \beta_{1}^{2} P_{\mathrm{s}} \boldsymbol{h}^{\mathrm{H}}\left(\theta_{u_{j}}\right) \boldsymbol{v}_{i} \boldsymbol{v}_{i}^{\mathrm{H}} \boldsymbol{h}\left(\theta_{e_{m}}\right)+\beta_{2}^{2} P_{\mathrm{s}} \boldsymbol{h}^{\mathrm{H}}\left(\theta_{u_{j}}\right) \boldsymbol{W} \boldsymbol{W}^{\mathrm{H}} \boldsymbol{h}\left(\theta_{u_{j}}\right)}\right)
\end{gathered}
$$




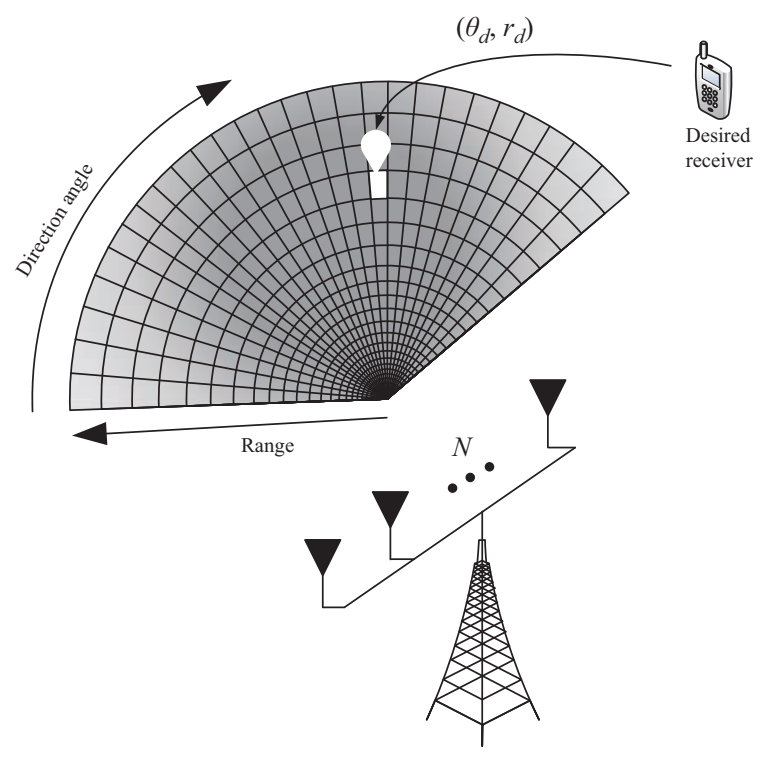

图 6 精准无线通信系统示意图

Figure 6 Schematic diagram of precision wireless communication system

因此, 安全速率和定义为主信道的信道容量减去最大的窃听信道容量:

$$
R_{\mathrm{s}}=\sum_{m=1}^{M}\left[R_{m}\left(\theta_{d_{m}}\right)-\max _{j} R_{m}\left(\theta_{u_{j}}\right)\right]^{+},
$$

$(x)^{+}$表示当 $x<0$ 时, $x=0$, 否则等于 $x$.

\section{4 基于方向调制的精准无线通信}

目前的大多文献研究工作中, 期望方向与非期望方向独立, 对于方向角相同的接收机和窃听机, 传 统的方向调制技术无法继续保证信息传输的安全性, 因其角度依赖性的特点, 波束指向在距离向是恒 定不变的. 如图 6 所示, 扇形亮区域即为指定的距离和角度位置, 其他则为窃听区域, 基于方向调制的 精准无线通信则希望能够将有用信号发射到指定的方向和距离附近小的邻域, 而在除此之外的其他区 域几乎不能接收到有用信号, 而是受到人工噪声的极大影响, 频率分集阵列 (又称频控阵) 发射波束恰 恰具有距离 - 角度依赖性的特点 ${ }^{[26,27]}$. 与相控阵类似, 频控阵列需附加很小的频偏 $\Delta f$ (远远小于载 频 $f_{0}$ ) 控制, 辐射的信号频率中心有所偏移, 但主要频率成分是重叠的.

下面以第一个阵元为参考, 第 $n$ 个阵元的发射频率表示为

$$
f_{n}=f_{0}+(n-1) \Delta f, \quad n=1,2, \ldots, N,
$$

令 $\Delta f_{n}=(n-1) \Delta f, f_{0}=f_{1}$. 假设每个阵元发射的信号相同, 只是阵元频偏不同, 设第 $n$ 个阵元的发 射信号表示为

$$
u_{n k}(t)=p_{n} q_{n k} \mathrm{e}^{-\mathrm{j} 2 \pi f_{n} t},
$$

其中, $p_{n}$ 表示第 $n$ 个阵元的激励信号向量成分, 并定义为 $p_{n}=\mathrm{e}^{\mathrm{j} \phi_{n}}, \phi_{n}$ 为第 $n$ 个阵元的初始相位, $q_{n k}$ 表示发送的第 $n$ 个阵元的第 $k$ 个调制复信息数据, 满足 $\mathrm{E}\left\{q_{n k}^{*} q_{n k}\right\}=1$. 传播到远场位置 $(\theta, r)$ 的 
信号表示为

$$
u_{n k}(\theta, r, t)=p_{n} q_{n k} \mathrm{e}^{-\mathrm{j} 2 \pi f_{n}\left(t-\frac{r_{n}}{c_{0}}\right)},
$$

式中, $\mathrm{c}_{0}$ 为光速, $r_{n}=r-(n-1) d \cos \theta$ 为第 $n$ 个阵元与远场目标 $(\theta, r)$ 之间的距离, 其中 $d$ 为阵元间 距. 则目标位置 $(\theta, r)$ 观察的波束图函数可表示为

$$
\begin{aligned}
\operatorname{AF}(t ; \theta, r) & =\sum_{n=1}^{N} u_{n k}(\theta, r, t)=\sum_{n=1}^{N} \mathrm{e}^{\mathrm{j} \phi_{n}} q_{n k} \exp \left\{-\mathrm{j} 2 \pi\left(f_{0}+\Delta f_{n}\right)\left(t-\frac{r-(n-1) d \cos \theta}{\mathrm{c}_{0}}\right)\right\} \\
& =\mathrm{e}^{-\mathrm{j} \varphi_{r}} \sum_{n=1}^{N} \mathrm{e}^{\mathrm{j} \phi_{n}} q_{n k} \exp \left\{-\mathrm{j} 2 \pi\left((n-1) \frac{f_{0} d \cos \theta}{\mathrm{c}_{0}}+\Delta f_{n}\left(t-\frac{r-(n-1) d \cos \theta}{\mathrm{c}_{0}}\right)\right)\right\},
\end{aligned}
$$

其中 $\varphi_{r}=2 \pi f_{0}\left(t-\frac{r}{c_{0}}\right)$. 发射导向向量为

$$
\boldsymbol{h}(\theta, r)=[\underbrace{\mathrm{e}^{\mathrm{j} 2 \pi \Delta \psi_{0}}}_{h_{1}(\theta, r)}, \underbrace{\mathrm{e}^{\mathrm{j} 2 \pi \Delta \psi_{1}}}_{h_{2}(\theta, r)}, \cdots, \underbrace{\mathrm{e}^{\mathrm{j} 2 \pi \Delta \psi_{N-1}}}_{h_{N}(\theta, r)}]^{\mathrm{T}},
$$

其中, $\Delta \psi_{n-1}$ 定义为

$$
\begin{gathered}
\Delta \psi_{n-1}=\psi_{n}-\psi_{0}, \quad n=1,2, \ldots, N \\
\psi_{n}=\frac{f_{n} r_{n}}{\mathrm{c}_{0}}, \psi_{0}=\frac{f_{0} r}{\mathrm{c}_{0}} .
\end{gathered}
$$

位于 $(\theta, r)$ 位置处的接收机接收到的信号表示为

$$
\begin{aligned}
y(\theta, r, t)= & \sum_{n=1}^{N} h_{n}^{*}(\theta, r) \mathrm{e}^{\mathrm{j} \phi_{n}} q_{n k} \exp \left\{-\mathrm{j} 2 \pi\left(f_{0}+\Delta f_{n}\right)\left(t-\frac{r-(n-1) d \cos \theta}{\mathrm{c}_{0}}\right)\right\} \\
& +\sum_{n=1}^{N} h_{n}^{*}(\theta, r) w_{n}+n(\theta, r) \\
= & \mathrm{e}^{-\mathrm{j} \varphi_{r}} \sum_{n=1}^{N} h_{n}^{*}(\theta, r) \mathrm{e}^{\mathrm{j} \phi_{n}} q_{n k} \exp \left\{-\mathrm{j} 2 \pi\left((n-1) \frac{f_{0} d \cos \theta}{\mathrm{c}_{0}}+\Delta f_{n}\left(t-\frac{r-(n-1) d \cos \theta}{\mathrm{c}_{0}}\right)\right)\right\} \\
& +\sum_{n=1}^{N} h_{n}^{*}(\theta, r) w_{n}+n(\theta, r),
\end{aligned}
$$

其中, $w_{n}$ 表示归一化人工噪声向量成分, $n(\theta, r)$ 表示接收噪声, 其分布满足 $n \sim \mathcal{C N}\left(0, \sigma_{\theta, r}^{2}\right)$. 对于人 为噪声的设计

$$
\boldsymbol{h}^{\mathrm{H}}\left(\theta_{d}, r_{d}\right) \boldsymbol{w}=\mathbf{0}
$$

通过正交投影的方法求出

$$
\boldsymbol{w}=\left(\boldsymbol{I}_{N}-\boldsymbol{h}\left(\theta_{d}, r_{d}\right)\left[\boldsymbol{h}^{\mathrm{H}}\left(\theta_{d}, r_{d}\right) \boldsymbol{h}\left(\theta_{d}, r_{d}\right)\right]^{-1} \boldsymbol{h}^{\mathrm{H}}\left(\theta_{d}, r_{d}\right)\right) \boldsymbol{z},
$$

因此

$$
\boldsymbol{h}^{\mathrm{H}}\left(\theta_{d}, r_{d}\right) \boldsymbol{w}=\boldsymbol{h}^{\mathrm{H}}\left(\theta_{d}, r_{d}\right)\left(\boldsymbol{I}_{N}-\boldsymbol{h}\left(\theta_{d}, r_{d}\right)\left[\boldsymbol{h}^{\mathrm{H}}\left(\theta_{d}, r_{d}\right) \boldsymbol{h}\left(\theta_{d}, r_{d}\right)\right]^{-1} \boldsymbol{h}^{\mathrm{H}}\left(\theta_{d}, r_{d}\right)\right) \boldsymbol{z}=0 .
$$




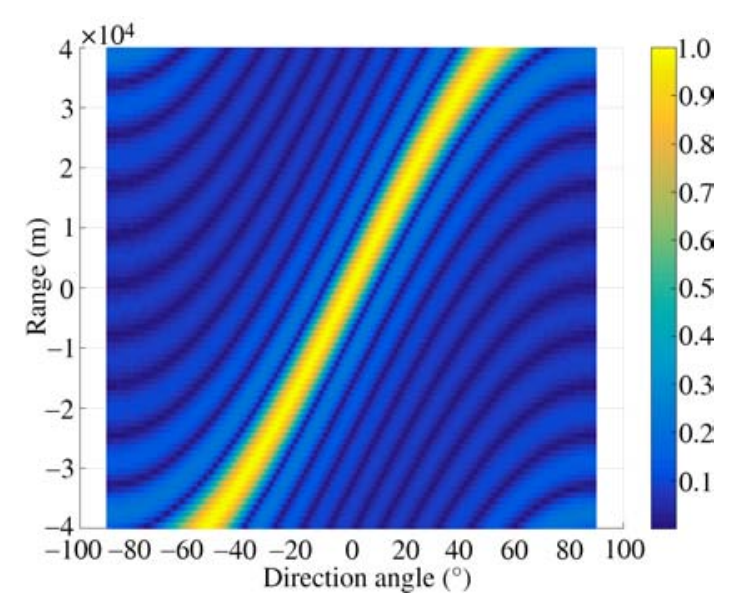

图 7 (网络版彩图) 常规 FDA 波束图

Figure 7 (Color online) Conventional FDA beam pattern

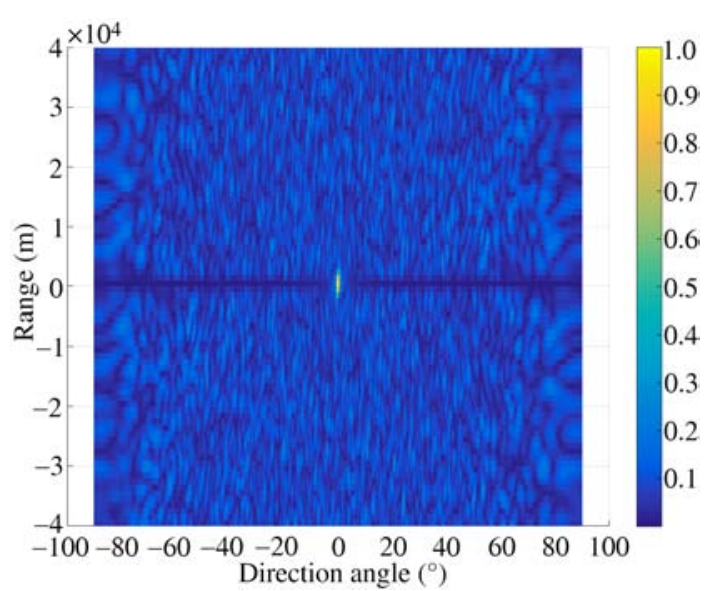

图 8 (网络版彩图) 距离 - 角度依赖性 FDA 波束图

Figure 8 (Color online) Range-angle-dependent FDA beam pattern

位于期望位置 $\left(\theta_{d}, r_{d}\right)$ 的接收机, 其接收信号可表示为

$$
\begin{aligned}
y\left(\theta_{d}, r_{d}\right)= & \mathrm{e}^{-\mathrm{j} \varphi_{r_{d}}} \sum_{n=1}^{N} h_{n}^{*}\left(\theta_{d}, r_{d}\right) \mathrm{e}^{\mathrm{j} \phi_{n}} q_{n k} \\
& \cdot \exp \left\{-\mathrm{j} 2 \pi\left((n-1) \frac{f_{0} d \cos \theta_{d}}{\mathrm{c}_{0}}+\Delta f_{n}\left(t-\frac{r_{d}-(n-1) d \cos \theta_{d}}{\mathrm{c}_{0}}\right)\right)\right\}+n\left(\theta_{d}, r_{d}\right) .
\end{aligned}
$$

位于非期望位置 $\left(\theta_{u}, r_{u}\right)$ 的接收机, 其接收信号可表示为

$$
\begin{aligned}
y\left(\theta_{u}, r_{u}\right)= & \mathrm{e}^{-\mathrm{j} \varphi_{r_{u}}} \sum_{n=1}^{N} h_{n}^{*}\left(\theta_{u}, r_{u}\right) \mathrm{e}^{\mathrm{j} \phi_{n}} q_{n k} \\
& \cdot \exp \left\{-\mathrm{j} 2 \pi\left((n-1) \frac{f_{0} d \cos \theta_{u}}{\mathrm{c}_{0}}+\Delta f_{n}\left(t-\frac{r_{u}-(n-1) d \cos \theta_{u}}{\mathrm{c}_{0}}\right)\right)\right\} \\
& +\boldsymbol{h}^{\mathrm{H}}\left(\theta_{u}, r_{u}\right) \boldsymbol{w}+n\left(\theta_{u}, r_{u}\right),
\end{aligned}
$$

式 (40) 中, 由于人为噪声位于期望位置导向向量的零空间, 而与非期望位置导向向量不正交, 因而 $\boldsymbol{h}^{\mathrm{H}}\left(\theta_{u}, r_{u}\right) \boldsymbol{w}$ 不为零, 从而严重污染窃听机对有用信号的接收, 实现精准无线传输的目的.

为便于仿真, 阵列各阵元初始相位由期望位置 $\left(\theta_{d}, r_{d}\right)$ 对应的导向向量设置: $h_{n}^{*}\left(\theta_{d}, r_{d}\right) \mathrm{e}^{\mathrm{j} \phi_{n}}=1$, 同 时可置 $t=0$. 如图 7 所示, 即为常规 FDA (frequency diversity array) 波束图, 频率增量 $\Delta f$ 固定, 取 $\Delta f=3 \mathrm{kHz}$. 图 8 则是精准无线传输波束图, 其中天线数为 128 , 第 $n$ 个阵元的频率增量 $\Delta f_{n}$ 为随机 值, 这里取 $\Delta f_{n}=\log (n) \cdot 30 \mathrm{kHz}$. 仿真结果可以看出: 即使窃听机的方向角与期望接收机的方向角相 同, 但各自与发射机的距离不同, 波束图的形成存在距离 - 角度维差异, 从而形成距离 - 角度依赖性 发射接收波束. 同时, 由于人为噪声的影响, 窃听机的接收性能下降, 从而确保无线传输的安全性.

\section{5 未来研究亟待解决的关键问题}

物理层安全技术为无线通信的安全提供保障, 其未来研究仍面临许多待解决的问题, 如方向调制 
在多径环境下的安全传输问题、信息论问题和精准无线通信在二维 (距离和方向角) 影响因素下的关 键技术以及其在估计误差条件下的鲁棒性问题.

方向调制在多径环境 (NLOS, No line-of-sight) 传播问题. 在无线通信中, 大气层对电磁波的 散射、周围建筑物等地表物体对电波的反射或绕射等都会造成无线电信号的多径传播. 如图 9 所示, 沿方向角 $\theta_{d}$ 的直达路径 (LOS, line-of-sight) 主要将有用信号传递到期望接收机, 而沿其他方向角, 如 $\theta_{1}$ 的路径会带有大量的人工噪声, 根据式 (7) 和 (8), 期望接收机端接收的信号表示为

$$
\begin{aligned}
y= & \underbrace{\{\underbrace{\beta_{1} \sqrt{P_{\mathrm{s}}} \boldsymbol{h}^{\mathrm{H}}\left(\theta_{d}\right) \boldsymbol{v} x}_{\text {Useful signals of LOS }}+\underbrace{\beta_{2} \sqrt{P_{\mathrm{s}}} \boldsymbol{h}^{\mathrm{H}}\left(\theta_{d}\right) \boldsymbol{W} \boldsymbol{z}}_{\mathrm{AN}_{\mathrm{d}}}\}}_{\text {LOS }} \\
& +\underbrace{\{\underbrace{\eta \cdot \beta_{1} \sqrt{P_{\mathrm{s}}} \boldsymbol{h}^{\mathrm{H}}\left(\theta_{1}\right) \boldsymbol{v} x}_{\text {Useful signals }}+\underbrace{\eta \cdot \beta_{2} \sqrt{P_{\mathrm{s}}} \boldsymbol{h}^{\mathrm{H}}\left(\theta_{1}\right) \boldsymbol{W} \boldsymbol{z}}_{\text {AN }}\}}_{\text {NLOS }}+\underbrace{\omega_{d, 1}}_{\text {AWGN }},
\end{aligned}
$$

在方向角完美已知的条件下，以正交投影法为例，上式中, $\boldsymbol{v}$ 和 $\boldsymbol{W}$ 参考式 (9) 和 (10), 根据式 (11), 式 (41) 可重新表示为

$$
y=\underbrace{\beta_{1} \sqrt{P_{\mathrm{s}}} \boldsymbol{h}^{\mathrm{H}}\left(\theta_{d}\right) \boldsymbol{v} x}_{\text {Useful signals of LOS }}+\underbrace{\eta \cdot \beta_{1} \sqrt{P_{\mathrm{s}}} \boldsymbol{h}^{\mathrm{H}}\left(\theta_{1}\right) \boldsymbol{v} x}_{\text {Useful signals }}+\underbrace{\eta \cdot \beta_{2} \sqrt{P_{\mathrm{s}}} \boldsymbol{h}^{\mathrm{H}}\left(\theta_{1}\right) \boldsymbol{W} \boldsymbol{z}}_{\text {AN }}+\underbrace{\omega_{d, 1}}_{\text {AWGN }},
$$

等式右边第一项为直达路径传输的信号, 人工噪声与期望方向导向向量正交, 因此直达路径信号主要 为有用信号; 等式右边第二、三项为沿 $\theta_{1}$ 经反射等途径后到达期望接收机的非直达路径信号, $\eta \leqslant 1$ 为反射率系数, 发射机端沿 $\theta_{1}$ 方向加入了人工噪声, 以扰乱对应方向上窃听者对有用信号的接收, 由 于 $v$ 位于期望方向导向向量空间, 则

$$
\begin{aligned}
\boldsymbol{h}^{\mathrm{H}}\left(\theta_{1}\right) \boldsymbol{v} & =\frac{1}{N}\left[\mathrm{e}^{-\mathrm{j} 2 \pi \varphi_{\theta_{1}}(1)}, \mathrm{e}^{-\mathrm{j} 2 \pi \varphi_{\theta_{1}}(2)}, \ldots, \mathrm{e}^{-\mathrm{j} 2 \pi \varphi_{\theta_{1}}(N)}\right]\left[\mathrm{e}^{\mathrm{j} 2 \pi \varphi_{\theta_{d}}(1)}, \mathrm{e}^{\mathrm{j} 2 \pi \varphi_{\theta_{d}}(2)}, \ldots, \mathrm{e}^{\mathrm{j} 2 \pi \varphi_{\theta_{d}}(N)}\right]^{\mathrm{T}} \\
& =\frac{1}{N \mathrm{e}^{\mathrm{j} 2 \pi \frac{(N+1) d}{2 \lambda}\left(\cos \theta_{d}-\cos \theta_{1}\right)}} \sum_{n=1}^{N} \mathrm{e}^{\mathrm{j} 2 \pi \frac{d}{\lambda}\left(\cos \theta_{d}-\cos \theta_{1}\right) n} \\
& =\frac{1}{N \mathrm{e}^{\mathrm{j} 2 \pi \frac{N d}{2 \lambda}\left(\cos \theta_{d}-\cos \theta_{1}\right)}} \frac{1-\mathrm{e}^{\mathrm{j} 2 \pi \frac{d}{\lambda}\left(\cos \theta_{d}-\cos \theta_{1}\right) N}}{1-\mathrm{e}^{\mathrm{j} 2 \pi \frac{d}{\lambda}\left(\cos \theta_{d}-\cos \theta_{1}\right)}},
\end{aligned}
$$

如果上式等于零, 即 $\boldsymbol{h}^{\mathrm{H}}\left(\theta_{1}\right) \boldsymbol{v}=0$, 意味着

$$
1-\mathrm{e}^{\mathrm{j} 2 \pi \frac{d}{\lambda}\left(\cos \theta_{d}-\cos \theta_{1}\right) N}=0,
$$

且

$$
1-\mathrm{e}^{\mathrm{j} 2 \pi \frac{d}{\lambda}\left(\cos \theta_{d}-\cos \theta_{1}\right)} \neq 0 .
$$

上面两式可以进一步简化为

$$
2 \pi \frac{d}{\lambda}\left(\cos \theta_{d}-\cos \theta_{1}\right) N=2 \pi m, \quad m \neq 0, \quad m \in \mathbb{Z}
$$




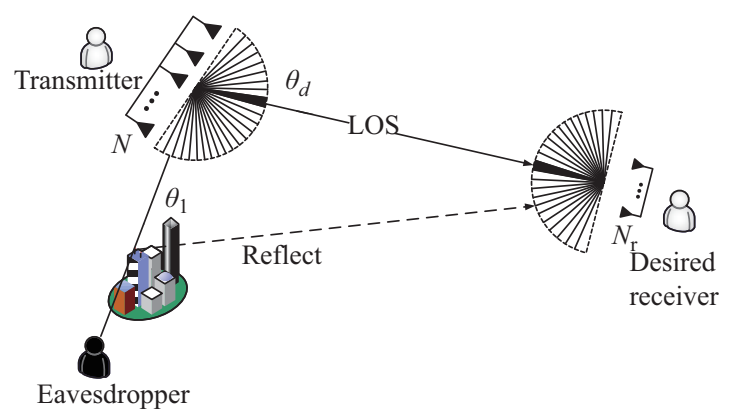

图 9 (网络版彩图) 多径传播示意图

Figure 9 (Color online) Schematic diagram of multi-path transmission

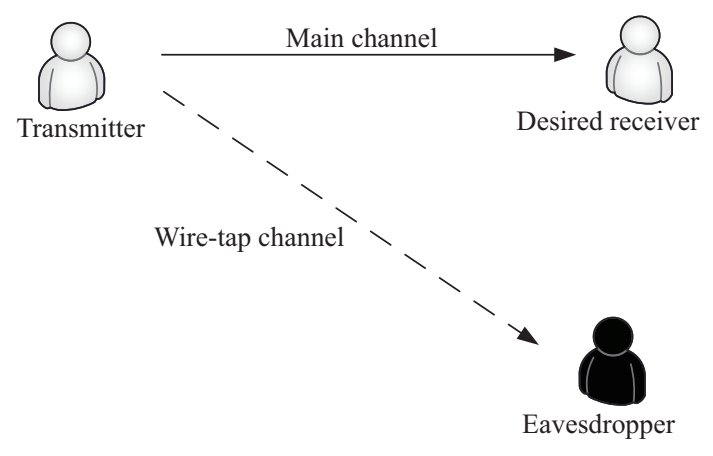

图 10 安全速率模型

Figure 10 Basic model of security rate

$$
m \in\left[\left\lceil\left(\frac{-d N\left(\cos \theta_{d}-\cos \theta_{1}\right)}{\lambda}\right),\left(\frac{d N\left(\cos \theta_{d}-\cos \theta_{1}\right)}{\lambda}\right)\right]\right]
$$

且

$$
\begin{gathered}
2 \pi \frac{d}{\lambda}\left(\cos \theta_{d}-\cos \theta_{1}\right) \neq 2 \pi m^{\prime}, \quad m^{\prime} \neq 0, \\
m^{\prime} \notin\left[\left[\left(\frac{-d\left(\cos \theta_{d}-\cos \theta_{1}\right)}{\lambda}\right),\left(\frac{d\left(\cos \theta_{d}-\cos \theta_{1}\right)}{\lambda}\right)\right]\right] .
\end{gathered}
$$

依据上述正交条件, 且给定 $\theta_{d}$ 条件下, 我们有

$$
\theta_{1}=\arccos \left(\cos \theta_{d}-\frac{m \lambda}{N d}\right),
$$

因此, 给定 $\theta_{d}, \theta_{1}$ 取上式值时, $\boldsymbol{h}^{\mathrm{H}}\left(\theta_{1}\right) \boldsymbol{v}=0, \boldsymbol{h}^{\mathrm{H}}\left(\theta_{1}\right)$ 与 $\boldsymbol{v}$ 正交时 (即 $\boldsymbol{h}^{\mathrm{H}}\left(\theta_{1}\right) \boldsymbol{v}=0$ ), 非直达路径传输的 信号将几乎全部是干扰有用信号的人工噪声, 该噪声信号与直达路径的有用信号叠加被期望接收机接 收, 由于接收到大量人工噪声, 加上接收噪声 (即等式右边第 4 项), 期望接收机接收到的有用信号受 到严重的污染, 性能严重恶化. 如何解决多径产生方向调制困境? 在使用发射天线阵列基础上, 引入 接收天线阵列, 运用接收波束成形应该是一种有效途径, 将主瓣对齐发射机, 从而剔除所有多径信号, 仅保留 LOS 路径. 同时可以考虑增加发射机天线阵列天线数目, 即采用大规模 MIMO 技术, 配置大 量天线, 将发射波束对准接收机接收波束, 进一步提高其传输信道的空间分辨率, 分离出直达路径, 减 少甚至完全消除多径的影响. 此也衍生出该系统关键技术波束对齐技术, 如何实现精确的波束对齐是 方向调制系统能否对抗多径的至关重要的关键技术之一.

信息论问题. 多用户场景下, 从信息论角度出发, 研究多用户系统安全容量区域问题、安全速率 和的渐进上界问题. 如图 10 所示为安全速率模型, 多期望用户安全速率将组成安全速率向量, 各个用 户安全速率会耦合其他用户干扰, 寻找最优的安全速率向量非常困难, 能综合考虑多用户利益, 设计出 Pareto 最优的波束成形算法更符合实际需求. 功率分配问题, 给定总的发射功率, 最大化安全速率和 是一个非凸优化问题, 我们可以将其转化为: 给定安全速率条件下, 最小化发射功率更易于求解. 异于 传统功率分配, 此处功率分配不仅包括多个期望用户功率分配, 还要考虑人为噪声功率分配问题, 是 一个非常具有挑战性的难题.

精准无线通信的基本理论与关键技术. 精准无线通信是一个全新的概念, 刚刚起步阶段, 许多基 本理论与关键技术问题急需深入探讨与研究, 譬如该系统的安全容量向量区域, 安全速率和的渐进上 
界, 如何设计稳健的波束成形方法, 如何实现最优的功率分配, 如何实现精确的波束对齐等等, 下面针 对功率分配方面, 提出可以开展研究的问题:

(1) 最大化安全速率: 在发射功率一定的情况下, 将有用信号的功率集中到指定的距离和方向位 置, 同时消除人工噪声干扰, 而窃听位置接收最大化的人工噪声功率, 从而最大化期望位置的信噪比以 提高期望用户位置的信息速率, 同时由于人工噪声的干扰使得窃听位置的信息速率最小化, 以获得最 大的安全速率为目的设计波束成形向量和人工噪声, 验证安全容量区间的推导.

(2) 最小化发射功率: 该算法设计方案可以转化为一个优化问题, 优化目标为最小化发射机端信号 的发射功率, 以提高功率利用效率, 但要满足一定的约束: (a) 期望用户的接收信噪比要大于所设置的 目标 SNR 阈值; (b) 非期望用户的接收信噪比要低于窃听 SNR 阈值, 可以考虑利用半定松弛和 KKT 条件优化得到波束成形向量和人工噪声协方差矩阵全局最优解 ${ }^{[23]}$.

另外, 实现波束成形需要知道用户的位置信息, 即方向与距离信息, 然而方向角和距离的估计会产 生各自的误差, 这些误差会恶化精准无线传输性能, 如此需要高精度的方向与距离估计算法, 或者高 性能定位算法.

\section{6 结束语}

本文总结了方向调制技术的基本原理、特点和应用, 综述了国内外关于方向调制的最新参考文献 与研究成果, 系统地阐述了方向调制的概念、基本理论以及关键技术, 深入探讨其未来需要解决开放 的关键问题, 并提供一些可行的解决思路, 进而提出整合方向调制和频率分集的精准无线传输全新概 念, 该技术可实现隐私信息安全传输, 可将隐私信息精准投送到空间指定的方向与距离附近小邻域, 不 会泄露到其他空间区域. 方向调制系列技术未来可应用于中继无线协作通信网络、无人机组网通信以 及卫星通信等多种场景, 因此具有广泛的应用前景和极其重要的理论研究意义.

\section{参考文献}

1 Csiszar I, Korner J. Broadcast channels with confidential messages. IEEE Trans Inform Theory, 1978, 24: 339-348

2 Diffie W, Hellman M. New directions in cryptography. IEEE Trans Inform Theory, 1976, 22: 644-654

3 Shiu Y S, Chang S Y, Wu H C, et al. Physical layer security in wireless networks: a tutorial. IEEE Trans Inform Theory, 2011, 18: 66-74

4 Hong Y W P, Lan P C, Kuo C C J, et al. Signal Processing Approaches to Secure Physical Layer Communications. Berlin: Springer, 2013

5 Wyner A D. The wire-tap channel. Bell Syst Tech J, 1975, 54: 1355-1387

6 Babakhani A, Rutledge D B, Hajimiri A. Transmitter architectures based on near-field direct antenna modulation. IEEE J Solid-State Circ, 2008, 43: 2674-2692

7 Babakhani A, Rutledge D, Hajimiri A. Near-field direct antenna modulation. IEEE Microw Mag, 2009, 10: 36-46

8 Daly M, Bernhard J T. Directional modulation technique for phased arrays. IEEE Trans Antenn Propag, 2009, 57: 2633-2640

9 Daly M, Daly E L, Bernhard J T. Demonstration of directional modulation using a phased array. IEEE Trans Antenn Propag, 2010, 58: 1545-1550

10 Daly M, Bernhard J T. Beam-steering in pattern reconfigurable arrays using directional modulation. IEEE Trans Antenn Propag, 2010, 58: 2259-2265 
11 Hong T, Song M Z, Liu Y. Dual-beam directional modulation technique for physical-layer secure communication. IEEE Trans Anrenn Propag, 2011, 10: 1417-1420

12 Hong T, Song M Z, Liu Y. Design of directional modulation signal based on multi-objective genetic algorithm for physical layer secure communication. J Appl Sci, 2014, 32: 51-56 [洪涛, 宋茂忠, 刘渝. 多目标遗传算法方向调制物 理层安全通信信号设计. 应用科学学报, 2014, 32: 51-56]

13 Ding Y, Fusco V. A vector approach for the analysis and synthesis of directional modulation transmitters. IEEE Trans Antenn Propag, 2014, 62: 361-370

14 Ding Y, Fusco V. Orthogonal vector approach for synthesis of multi-beam directional modulation transmitters. IEEE Antenn Wirel Propag Lett, 2015, 14: 1330-1333

15 Ding Y, Fusco V. MIMO inspired synthesis of directional modulation systems. IEEE Antenn Wirel Propag Lett, 2015, 15: $580-584$

16 Hafez M, Arslan H. On directional modualtion: an analysis of transmission scheme with multiple directions. In: Proceedings of the IEEE International Conference on Communications, London, 2015. 459-463

17 Zhao N, F Richard Yu, Li M, et al. Physical layer security issues in interference-alignment-based wireless networks. IEEE Commun Mag, 2016, 54: 162-168

$18 \mathrm{Hu}$ J S, Shu F, Li J. Robust synthesis method for secure directional modulation with imperfect direction angle. IEEE Commun Lett, 2016, 20: 1084-1087

19 Shu F, Wu X M, Li J, et al. Robust beamforming scheme for secure multi-beam directional modulation in broadcasting systems. IEEE Access, 2016, 4: 6614-6623

20 Sadek M, Tarighat A, Sayed A H. A leakage-based procoding scheme for downlink multi-user mimo channels. IEEE Trans Wirel Commun, 2007, 6: 1711-1721

21 Stoica P, Moses R L. Spectral Analysis of Signals. 3rd ed. New York: McGraw-Hill, 2006

22 Horn R A, Johnson C R. Matrix Analysis. Bridge: Cambridge University Press, 1987

23 Liao W C, Chang T H, Ma W K, et al. Joint transmit beamforming and artificial noise design for Qos discrimination in wireless downlink. In: Proceedings of the IEEE International Conference on Acoustics, Dallas, 2010. 2562-2565

24 Kraus J D, Marhefka R J. Antennas for All Applications. 3rd ed. New York: McGraw-Hill, 2006

25 Proakis J G. Digital Communications. New York: McGraw-Hill, 1983

$26 \mathrm{Hu}$ J S, Yan S H, Shu F, et al. Artificial-noise-aided secure transmission with directional modulation based on random frequency diverse arrays. IEEE Access, 2017, 5: 1658-1667

27 Wang W Q. Subarray-based frequency diverse array radar for target range-angle estimation. IEEE Trans Aerosp Electron Syst, 2014, 50: 3057-3067

\title{
Directional modulation-based secure wireless transmission: basic principles, key techniques, and applications
}

\author{
Feng SHU ${ }^{1,2,3,4}$, Xiaomin WU ${ }^{1}$, Xiaohu YOU ${ }^{3}$, Jinhui $\mathrm{LU}^{1 *}$, Jinsong HU${ }^{1}$, Wei ZHU ${ }^{1}$, Hai YU ${ }^{1}$, \\ Zhengwen $\mathrm{XU}^{2} \&$ Riqing $\mathrm{CHEN}^{4}$ \\ 1. School of Electronic and Optical Engineering, Nanjing University of Science and Technology, Nanjing 210094, \\ China; \\ 2. National Key Laboratory of Electromagnetic Environment, China Research Institute of Radiowave \\ Propagation, Qingdao 266107, China; \\ 3. National Mobile Communications Research Laboratory, Southeast University, Nanjing 210096, China; \\ 4. College of Computer and Information Sciences, Fujian Agriculture and Forestry University, Fuzhou 350002, \\ China \\ * Corresponding author. E-mail: lujinhui_ee_1962@163.com
}




\begin{abstract}
Directional modulation (DM), as an advanced physical layer security wireless transmission, will further improve the performance of the communication system by combining beamforming and artificial noise (AN). Unlike traditional beamforming systems, a DM system can transmit confidential messages along the predefined desired direction to enhance the safety in the desired direction and simultaneously disrupt the signal constellation pattern at undesired receivers. In this paper, the concept of DM, its principle, and application characteristics are described. Furthermore, implementation schemes and performance matrices of DM are systematically expounded. Finally, the research works on DM technology are summarized, and the future research potential trends are analyzed, along with several technical challenges. To solve the problem of angle-dependent transmit beam-pattern encountered in traditional DM, we propose a new secure wireless transmission concept named precision wireless transmission that combines DM and frequency diversity. This transmission technology can send confidential messages to a small neighborhood of the given arbitrary angle and range dimension without leakage to other regions.
\end{abstract}

Keywords directional modulation, physical layer security, artificial noise, beamforming, precision communication

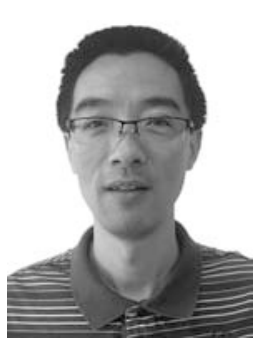

Feng SHU was born in 1973. He received the Ph.D., M.S., and B.S. degrees from the Southeast University, Nanjing, in 2002, Xidian University, Xi'an, China, in 1997, and Fuyang Teaching College, Fuyang, China, in 1994, respectively. In October 2005, he joined the School of Electronic and Optical Engineering, Nanjing University of Science and Technology, Nanjing, China, where he is currently a professor and supervisor of Ph.D and graduate students. His research interests include wireless networks, wireless location, and array signal processing.

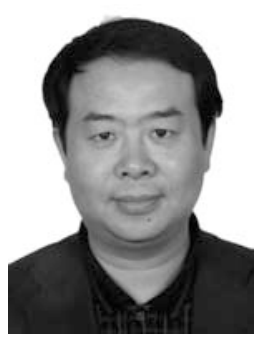

Xiaohu YOU was born in 1962. He received his Master's and Ph.D. degree from Southeast University, Nanjing, China, in electrical engineering in 1985 and 1988, respectively. Since 1990, he has been working with the National Mobile Communications Research Laboratory at Southeast University, where he is a professor since 1992, Changjiang Scholar program professor since 2000, and director since 2002. His research interests include wireless transmission, wireless networking, and signal processing and its applications.

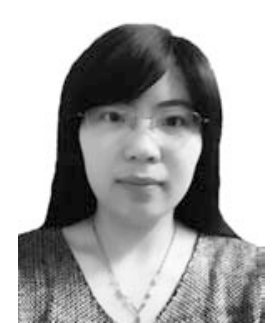

Xiaomin WU was born in 1992. She received the B.S. degree at Nanjing University of Science and Technology, Nanjing, China, in 2015. Now, she is with the School of Electronic and Optical Engineering, Nanjing University of Science and Technology, China and pursues her M.S. degree from the university. Her research interests include wireless communication and mobile networks.

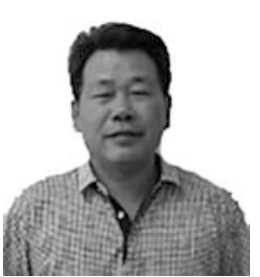

Jinhui LU was born in 1962 . He received the M.S. degree in communication and information systems from Nanjing University of Science and Technology, Nanjing, China, in 1985. He is currently a professor in the School of Electronic and Optical Engineering at Nanjing University of Science and Technology. His research interests including millimeter-wave monitoring systems, echo simulation of radar, and passive location technology. 